V.

\title{
Ueber Incarceratio stercoralis, die Kothverstopfung der Brïche (der angeblich erste Grad der Einklemmung).
}

Von

Prof. Dr. Fdm. Rose.

Zur Beurtheilung des Gegensatzes, wie er zwischen Darmeinklemmung (Incarceration) und Darmversehluss (Ileus) besteht, ist besonders lebrreich der lleus, welcher durch grosse Brüche veranlasst wird. Bei der Einklemmung handelt es sich um eine Ernährungsstörung eines Darmstickes durch Unterbrechung seiner Blutzufuhr, welche zunächst eine Gasauftreibung des Darmes - man nennt dies jetzt „localen Meteorismus" - und demnäehst Brand der Einklemmungsfurche zur Folge hat. Brand des eingeklemmten Darmstickes mit Collaps oder Ausgang in allgemeine Bauchfellentzundung pflegt dann das Ende zu sein, wenn nicht rechtzeitig eingegriffen ist. ${ }^{1}$ )

Beim Darmverschluss handelt es sich um die Staung des Darminhaltes, meist infolge eines allmählich wachsenden Hindernisses, welches wohl zuletzt zum Kothbrechen und zum Tod dureh Erschöpfung, aber niemals zum Darmbrand fubrt, wenn seine Beseitigung nicht gelingt.

Während bei der Einklemmung das Hauptzeichen die spontane Antiperistaltik des zufürenden Darmtheiles, Erbrechen oder wenigstens Aufstossen und Uebelkeit sind, kann das ganz beim Ileus feblen. Meist sehen wir beim Ileus, wenn der Bauch noch so sehr auftreibt, Erbrechen erst dann erfolgen, wenn unpassende Nahrungsmittel zugeführt werden, welche die Verstopfung vollenden, oder bei der Anwendung von Abführmitteln, welche das Hinderniss zu tuberwinden nicht vermögen, aber dennoch die Antiperistaltik des Magendarm-

1) Es gehören dazu die Hernien, innern Einklemmungen, der Valvulus und auch die Intussusception. Vgl. darüber meine „Weitere Beobachtungen über den Bruchschnitt" in der deutschen Zeitschrift für Chirurgie von Lücke und Rose Bd. XXXV. Heft 1. 1892. S. 1. 
endes zu vermehren im Stande sind. Je länger sie dauert, je öfter sie wiederkehrt, desto mehr wird sich sowobl das Ende des zufuihrenden Darmes, als auch sein Inbalt verändern. Er kommt dann einmal zur Verdickung und Erweiterung des Darmes oberbalb des Darmverschlusses, andererseits entweder zur Verhärtung und Bildung förmlicher Kothsteine oder zur Zersetzung, wenn es sich nicht um sterile Massen, wie z. B. beim regulinischen Quecksilber handelt, mit ihren Folgen, der Geschwirsbildung und schliesslich Fistelbildung. Das ist ja der gewöhnliche Vorgang, wie er bei Proktitis stercoralis und der Bildung der Mastdarmfisteln auf der einen Seite, bei der Typhlitis stercoralis und der Wurmfortsatzperforation auf der anderen Seite, zu Grunde liegt.

Seitdem ich 1865 in meiner Habilitationsschrift, „den Beobachtungen über den Bruchschnitt", diesen Gegensatz besprochen und hervorgehoben ${ }^{1}$ ), habe ich 3 mal Gelegenheit gehabt, mich bei grossen Hernien durch die Obduction von diesem Gegensatz zu überzeugen. Gerade daraus, dass diese Fälle schon recht alt sind, scheint mir hervorzugehen, dass diese Vorkommnisse verhältnissmässig selten sich ereignen; auch sonst verdienen dieselben ihrer Eigenthümlichkeit wegen mitgetheilt zu werden. Besonders mag es aber desbalb geschehen, weil noch immer von manchen Autoren - ich erinnere nur an die letzte, achte Auflage von Bardeleben's Chirurgie von einer Kotheinklemmung der Brüche, Incarceratio stercoralis, neben der gewöhnlichen Einklemmung gesprochen oder sie gar als ibr erstes Stadium hingestellt wird!

Die ersten beiden Fälle, welche ich gesehen, betrafen alte, grosse Nabelbriche.

\section{Eine Hernia pancreatica.}

In dem ersten Fall handelte es sich um eine 64 Jabre alte Bäckermeisters Frau A., aus der Oranienstrasse in Berlin; gross und stark, hatte sie viele Kinder zur Welt gebracht. Lange nach der Geburt ihres letzten Kindes war der Nabelbruch erst entstanden, welcher jetzt 20 Jahre bei ihr bestand.

Am 9. Juni 1867 war sie unter Verdauungsbeschwerden mit Schmerzen im Bruch erkrankt, und wurde ich, als ich noch Privatdocent der Chirurgie in Berlin war, am 11. Juni von dem Hausarzt hinzugezogen. Auf dem mächtigen Nabelbruch befand sich noch ein kleiner, kartoffelgrosser, dessen Inhalt sich jedoch ganz leicht reponiren liess. An eine Operation war um so weniger zu denken, als Wilms zu der Zeit die Operation des eingeklemmten Nabelbruches selbst bei den besten Privat-

1) Berlin bei Otto Janke 1867. Besonderer Abdruck aus den Charité-Annalen. Bd. XIV. Heft 1 . 
verhältnissen und fruhzeitiger Zuziehung ihrer schlechten Resultate wegen ein für allemal ablehnte. Eine blühende junge Frau in seiner Privatpraxis hat sich danach freilich bis in die 2 . Woche gequält, ehe sie der Einklemmung erlag. Hier handelte es sich aber nicht etwa um Einklemmung, sondern nur um die sogenannte Kothverstopfung. Am 12. war ihr Puls schon auf 140 gestiegen; zu der Verstopfung war jetzt, nachdem man ihr ein Abftihrmittel gegeben, Erbrechen hinzugetreten. Ohne operative Intervention ging die wohlbeleibte Frau zu Grunde.

Bei meiner Section ergab sich, dass ihr Fettpolster iiber 1 Zoll stark, tiber dem Nabelbruch hingegen höchstens 2 Linien maass. Der mächtige, kugelförmige Nabelbruch war 11 Zoll breit und 8 Zoll hoch. Beim Aufschneiden des Bruches fand ich darin das Colon transversum und ganze ascendens mit langem Gekrös. Der Wurmfortsatz war mit der Spitze an den Bruchsack angewachsen, ebenso das Netz mit dem Colon und Sack verwachsen. Die Dünndarmschlingen, welche darin lagen, waren blass. Ausserdem fand sich darin merkwtirdiger Weise das Pankreas. Einige Dtinndarmschlingen im Bruch zeigten entziundliche Röthe, wie das auch bei anderen Schlingen, die sich beim Aufschneiden in der Bauchhöhle fanden, der Fall war. Ausserdem lagen in der Bauchhöhle Magen, Leber, Milz und Geschlechtstheile. Der Bruchsackhals befand sich zwischen den Muskeln. Nirgend war in der Bauchhöhle oder im Bruch etwas von Brand zu bemerken, auch war in der Bauchhöhle keine Spur von Flussigkeit, nirgends ein Eiterbeschlag zu finden.

Der Tod war also an Erschöpfung erfolgt.

Eine so aussergewöhnliche Verschiebung der Baucheingeweide findet sich sonst nur bei angeborenen Brtichen vor. Ich habe selbst fruber, 1867, einen Fall von über kindskopfgrossem Nabelschnurbruch geheilt, bei dem ausser kleinen Därmen sich ein grosser Theil der Leber in der Nabelschnur befunden batte. Später habe ich einmal Gelegenheit gehabt, die grossmächtige Nabelplatte, mit der die Heilung zu Stande gekommen war, am Präparat der geburtshülflichen Gesellschaft in Berlin zu demonstriren.')

Viel merkwürdiger ist aber dieser Fall noch deshalb, weil es sich hier um die allmähliche Herauszerrung eines retroperitonealen Organes handelt, nämlich des Pankreas. Ich pflegte von da ab in meinen Vorlesungen alljährlich diesen Fall von Hernia pancreatica bei der Bruchlehre zu besprechen, um zu zeigen, dass der Chirurg selbst unter damaligen Verhältnissen mit retroperitonealen Organen zu thun haben könnte. Dabei ist zu betonen, dass es sich bier um einen erworbenen Nabelbruch gehandelt hat, der sich im Laufe von

1) Vgl. meine Beiträge zur Kenntniss der angeborenen chirurgischen Krankheiten des Meschen XII. „Ueber die Heilung des Nabelschnurbruches“ S. 13, auch "Kasuistische Mittheilungen, vorgetragen in der Gesellschaft für Geburtshülfe in Berlin vom 7. A pril 1868" in der Monatsschrift für Geburtskunde und Frauenkrankheiten. Bd. XXXII. 
20 Jahren entwickelte. Ermöglicht wurde diese Herauszerrung eines retroperitonealen Organes wobl dadurch, dass das Colon mit der vorderen Seite des Brucbsackes verwachsen war, wohl eine Folge der exponirten Lage.

\section{Eine Hernia pylorica.}

Zu einem ganz ähnlichen Fall wurde ich am 25. Juni 1867 bei einer Frau in der Schönhauser Allee in Berlin zugezogen. Es handelte sich um einen 3 fachen Bauchbruch am Nabel, von denen der oberste tympanitisch besonders sehr schmerzhaft war. Die Krankheit war zu Stande gekommen durch eine Ueberladung des Magens. Am nächsten Tage hatte nach dem Verbrauch von 5 Gran Crotonöl in Oleum Ricini das Erbrechen nachgelassen. Immerhin waren bei demselben zwei Pfifferlinge vollständig unverändert zum Vorschein gekommen, von denen sie einige Tage zuvor eine sehr grosse Menge zu sich genommen hatte, ohne dies ibr Lieblingsgericht, wie es scheint, sonderlich zu kauen.

Am nächsten Tage lag sie sichtlich im Sterben. Bei der Section am 28. Juni 1867 fand ich in dem oberen Bauchbruch, welcher 8 Zoll breit und 7 Zoll hoch war, einen Theil des Magens nebst dem Colon adhärent. Der Magen war 19 Zoll lang und von demselben der 4 Zoll lange Pylorustheil im Bruch. Im unteren, 9 Zoll breiten und 8 Zoll hohen Bruchsack befand sich nur Netz und Dündarm, und an einer dünnen Stelle lagen dort die Pfifferlinge (ich glaube, der College bat 96 gezählt). Die Schlingen waren ungleich, zum Theil ganz, zum Theil nur gesprenkelt roth. Es fand sich kein Exsudat vor. Der Blinddarm lag in der Bauchhöhle selbst, das Netz war links angewachsen, wo anch die dilatirten und gesprenkelten Schlingen lagen, welche viel Flussigkeit enthielten. Nirgends war eine Spur von Brand zu bemerken.

So haben wir in dieser Hernia pylorica ein Gegenstück zur eben erwähnten Hernia pancreatica! Hier interessiren uns aber beide Fälle, weil sie zeigen, dass die Kothverstopfung der Bernien, selbst wenn sie zum Tode fuhrt, niemals in Einklemmung ubergeht. Der Tod erfolgt, ohne dass das wesentliche Zeichen der Einklemmung, der Brand, eintritt.

Der dritte Fall von Kothverstopfung in Hernien, welchen ich babe zum Tode fíbren seben, betraf angeblich „eine angeborene Hodensackgeschwulst bei einem Neugeborenen."

\section{Eine Incarceratio stercoralis congenita.}

Das Kind wurde mir von einem bekannten Chirurgen in Zurrich in der ersten Zeit meiner klinischen Laufbahn in den 60 er Jahren zur Operation in die Klinik unmittelbar vor ihrem Beginn gebracht. Da der Leistenkanal ganz frei zu sein sehien, und die kindskopfgrosse Geschwulst verschiedene steinharte Knollen an ihrer Oberfläche zeigte, so einigten wir uns tiber die Diagnose eines angeborenen Osteochondroms der Testis nacheiner ausfihrlichen Besprechung aller möglichen Tumoren dieser Gegend. 
Beim Blosslegen war ich nicht wenig betroffen, in der betreffenden Hodensackhälfte eine beträchtlich blasse Darmschlinge zu finden, welche sich um lauter wallnussgrosse, steinharte Knollen in ihrem Inneren rosenkranzartig angeschmiegt hatte. An eine Reposition war absolut nicht zu denken, ohne eine ganz ausgiebige Herniocoeliotomie vorauszuschicken, da die Knollen für den engen Leistenkanal viel zu gross waren. Unter diesen Umständen brach ich mit der Operation ab und verordnete warme Umschläge, um diese harten Knollen womöglich bis zum nächsten Tage weich zu bekommen. Allein am nächsten Tage war das Kind bei meinem Besuche der Klinik bereits ohne Weiteres verschieden.

Bei der Section fand Herr Professor Eberth keine Spur von Brand, Einklemmung oder Peritonitis, auch keine sonstige Bildungsanomalie, als diese merkwürdige Verlängerung vom Gekrös der Flexura sigmoidea, welche derselben gestattet hatte, sich in den Hodensack hinabzubegeben. Verwachsen war es dort nicht. Beim Aufschneiden des Darmes fiel einer von dem Dutzend Knollen auf das Ofenblech hinab, es klang wie der Fall einer Steinkngel. Es handelte sich um harte, schwer schneidbare Kothsteine, deren Inneres vollständig geschichtet war.

Dieser etwas räthselhafte Fall bätte uns alle bei Lebzeiten vielleicht nicht so in die Irre geführt, wenn wir nicht erst lange Zeit hinterher erfahren hätten, dass das Kind wenigstens einmal vor der Operation gebrochen bat, worauf freilich bei Neugeborenen nicht viel gegeben wird und auch zu geben ist.

Die ubrigen Fälle von Kothverstopfung bei den Scrotalbrilchen der Männer sind früher ohne Radicaloperation durchgekommen. Aus solchen Fällen habe ich schon damals (1865) geschlossen, dass zwischen Ileus und Incarceration ein Gegensatz besteht. Der wesentliche Unterschied beider ist die in Brand endigende Circulationsunterbrechung bei der Einklemmung. 